\title{
Inhibition of HES-1 might play a protective role in endothelial cells under cholesterol stimulation via PI3K/AKT signaling pathway
}

\author{
Yun $\mathrm{Shi}^{1, \star}$, Ya Su${ }^{2,3, *}$, Chuanli $\mathrm{Liu}^{4, \star}$, Jinglong $\mathrm{Tao}^{5}, \mathrm{Hao} \mathrm{Fu}^{6}$ and Chengzhi $\mathrm{Lu}^{7}$ \\ ${ }^{1}$ The Fourth Center Clinical College of Tianjin Medical University, Tianjin, China \\ ${ }^{2}$ Department of Neurosurgery, Characteristic Medical Center of Chinese People's Armed Police Force, Tianjin, China \\ ${ }^{3}$ Department of Neurosurgery, Pingjin Hospital, Tianjin, China \\ ${ }^{4}$ Obstetrics and gynecology department, Pingjin Hospital, Tianjin, China \\ ${ }^{5}$ Hospital of Hunan Chinese People's Armed Police Force, Hunan, China \\ ${ }^{6}$ Cadre's ward, Characteristic Medical Center of Chinese People's Armed Police Force, Tianjin, China \\ 7 Tianjin First Center Hospital of Tianjin Medical University, Tianjin, China
}

\begin{abstract}
The occurrence of atherosclerotic cardiovascular disease (ASCVD) was closely related to low-density lipoprotein (LDL) cholesterol. HES-1 is critical for maintains of stem cells, quiescent cells or cancer cells, and contributes to drug resistance and metastasis of tumor cells. In this study, we established a cell model of HES-1 inhibition and overexpression in Ea.hy 926 cells, and firstly detected the proliferation rete of Ea.hy 926 cells under cholesterol stimulation using MTT assay, and apoptosis of Ea.hy 926 cells were detected using flow cytometry. Expression of HES-1, apoptosis related proteins and phosphatidylinositol 3 kinase (PI3K)/protein kinase B (AKT) signaling pathway were detected using Western blotting analysis. The expression of apoptotis related genes were detected using polymerase chain reaction (PCR) method. The concentration of angiogenesis cytokines was detected using enzyme-linked immunosorbent assay (ELISA) method. We found that proliferation of Ea.hy 926 cells was inhibited after stimulation of cholesterol, inhibition of HES-1 expression would reduce this effect. We also found that expression of apoptosis related molecules was increased and expressions of angiogenesis factors were decreased after cholesterol treatment. Besides, we revealed that these effects were mediated via PI3K/AKT signaling pathway, and HES-1 inhibition could increase the activity of this signaling pathway.
\end{abstract}

Key words: HES-1 - Cholesterol - Cardiovascular disease - Endothelial cells - Apoptosis

\section{Introduction}

One of the leading causes of morbidity and mortality worldwide is atherosclerotic cardiovascular disease (ASCVD) (Goldstein et al. 2015). Previous study found the direct relationship between concentrations of low-density lipoprotein

\footnotetext{
* These authors contributed equally to this work.

Correspondence to: Hao Fu, Cadre's ward, Characteristic Medical Center of Chinese People's Armed Police Force, Tianjin, No.220 Chenglin Road, Dongli District, China

E-mail: 576910239@qq.com

Chengzhi Lu, Tianjin First Center Hospital of Tianjin Medical University, Tianjin, No.24 Fukang Road, Nankai District, China

E-mail: lucz8@126.com
}

(LDL)-cholesterol in plasma and the occurrence of cardiovascular disease. Dysfunction of endothelial cells might be a critical pathogenesis for cardiovascular disease, including hypertension and pregnancy-induced hypertension (Ference et al. 2017). Clinical trials proved that lipid-lowering drugs could significantly decrease the concentration of LDL-cholesterol, resulting in reduction in morbidity and mortality in cardiovascular disease patients and healthy people (Schwartz et al. 2018). Previous study indicated that transcriptional repressor of Hes family BHLH transcription factor 1 (HES-1) protects cells from differentiation, and some tumor cells even relay on HES- 1 to protect against differentiation, and down-regulation of HES-1 leads to the reduction of cellular proliferation in ovarian cancer cells (Sang et al. 2008; Majidinia et al. 2017). There is also study found that almost all of undifferentiated cells is expressed HES-1 (Kageyama et al. 2005). HES-1 de- 
ficient mice presented some severe defects in brain, eye and pancreas (Ishibashi et al. 1995), HES-1 expression is disappeared when cells initate differentiation. However, there is also study indicated that promote the differentiation of neuronal and beta cell through silencing the repressor element silencer transcription (REST) factor (Abderrahmani et al. 2005).

In present study, we firstly constructed a HES-1 inhibition and HES-1 overexpression model in Ea.hy 926 cells, and explored the function of HES-1 on proliferation of endothelial cells under cholesterol stimulation. We further explored the effect of HES-1 in altering the expression of apoptosis and angiogenesis related molecules at gene and protein levels. And we also found that this effect might be mediated by PI3K/AKT signaling pathway, while reduction of HES-1 expression would enlarge this effect.

\section{Material and Methods}

\section{Material}

Fetal bovine serum (FBS) (12484-028) and High Glucose Dulbecco's Modified Eagle's Medium (H-DMEM) (11965092) were purchased from Thermo (NY, USA). Cholesterol was purchased from Sigma (C4951). MTT Cell Proliferation and Cytotoxicity Assay Kit (C0009) were purchased from Beyotime (Shanghai, China). KpnI (R0142L), XhoI (R0146L), Quick Ligase (M2200S) and T4 PNK (M0201S) were purchased from NEB (NY, USA). FastDigest BsmBI (FD0454) was purchased from Fermentas (NY, USA). Hieff Trans transfection reagent (40802ES01) and G418 (60220ES03) were purchased from Yeasen Biotechnology Co (Shanghai, China). Protease Inhibitor Cocktail (CW2200), Ultrapure RNA Kit (CW0597) and Super TaqMan OneStep RT-qPCR Kit (CW2695) were purchased from CWbio Biological Technology Company (Beijing, China). Anti-HES-1 (ab108937), Wnt (ab15251), AKT (ab8805), p-AKT (ab38449), $\beta$-catenin (ab32572), c-Myc (ab32072), Bax (ab32503), Bcl-2 (ab196495), BIM (ab32158), p-BIM (ab17935), caspase-9 (ab32539), caspase-3 (ab13847) antibodies and TGF- $\beta$ (ab119558), EGF (ab239424), VEGF- $\alpha$ (ab100786), PDGF (ab155464) ELISA kits were purchased from Abcam. Ea.hy 926 cells (No. GNHu39) and 293T cells (SCSP-502) were obtained from the cell library of the typical culture preservation committee of Chinese academy of sciences.

\section{Vector construction}

cDNA of HES-1 was cloned from cells using PCR methods using the primers listed: Forward: 5'-TCTACACCAGCAACAGTGG-3'; Reverse: 5'-TCAAACATCTTTGGCATCAC-3'. Blank pCNDA3.1-3×Flag vector and PCR product of HES-1 were digested with KpnI and XhoI, then HES-1 fragment was cloned into vector to construct pCDNA3.1$3 \times$ Flag- HES- $1 \beta$ overexpression vector. Ea.hy 926 cells were transfected using pCDNA3.1-3×Flag- HES-1 vector using Hieff Trans transfection reagent for $48 \mathrm{~h}$ under the guidance of manufacturer's protocol. Stable HES-1 overexpression cells were screened using $1000 \mu \mathrm{g} / \mathrm{mL}$ G418. HES-1 knockdown vector was constructed according to previous study (Canver et al. 2018). Briefly, CRISPR vector was firstly digested with BsmBI according to the protocol, and annealed oligoes was constructed using primers listed: Forward: 5'-CACCGC CGCTTACGGCGGACTCCATG-3'; Reverse: 5'- AAACCATGGAGTCCGCCGTAAGCGC-3'. Then ligation reaction was performed using annealed oligoes and digested CRISPR vector according to the protocol of Quick Ligase to construct the HES-1 knockdown vector. The lentivirus vector was constructed using $293 \mathrm{~T}$ cells, and stable HES-1 knockdown cells were screened using $2 \mu \mathrm{g} / \mathrm{ml}$ puro.

\section{Cell culture and grouping}

Cells were cultured in H-DMEM supplied with 10\% FBS under a humid $37^{\circ} \mathrm{C}$ atmosphere supplied with $5 \% \mathrm{CO}_{2}$. Cells were grouped into four groups: control group (NC), cholesterol stimulation group $(\mathrm{CH})$, HES-1 overexpression with cholesterol stimulation group (GO) and HES-1 knockdown with cholesterol stimulation group (GD).

\section{Cellular toxicity of cholesterol on Ea.hy 926 cells}

Cells in different groups were stimulated with different concentration of cholesterol for different period. Briefly, cells were seeded into each well of a 96-well plate at a concentration of $1 \times 10^{4}$ and cultured until confluence of cells reached $80-85 \%$, cells in cholesterol stimulation groups were treated with $50 \mathrm{mmol} / \mathrm{l}, 100 \mathrm{mmol} / \mathrm{l}$ and $200 \mathrm{mmol} / \mathrm{l} \mathrm{cholesterol} \mathrm{for}$ 12, 24 and $48 \mathrm{~h}$. Cells were incubated with $5 \mathrm{mg} / \mathrm{ml} \mathrm{MTT}$ reagent for $4 \mathrm{~h}$ after washed with PBS, and then OD value at $560 \mathrm{~nm}$ was determined using SuPerMax 3100 microplate reader (Shanghai, China) after DMSO was added. Viability of cells $=\left(\mathrm{OD}_{\text {Experiment }}-\mathrm{OD}_{\text {Blank }} / \mathrm{OD}_{\text {Control }}-\mathrm{OD}_{\text {Blank }}\right)$ $\times 100 \%$.

\section{RNA extraction and reverse transcription}

RNA extraction was proceeded under the guidance of the protocol of Ultrapure RNA Kit. Briefly, cells were firstly lysed with TRIzol reagent followed with incubation at room temperature for $5 \mathrm{~min}$, then mixed with chloroform followed by vigorous shake for 15 s. Then $70 \%$ ethanol was added after centrifuged for $5 \mathrm{~min}$ at $12,000 \mathrm{rpm}$, and then mixture was loaded onto an adsorption column and centrifuged for $1 \mathrm{~min}$ at 12,000 rpm. After washed with wash buffer, RNA 
adsorbed in the adsorption column was eluted using elution buffer. Concentration of RNA was detected using Nanodrop ND-2000 (Thermo Fisher Scientifc, Inc.).

Reverse transcription and real-time quantitative polymerase chain reaction ( $q P C R$ )

Reverse transcription and qPCR were performed according to the protocol of Super TaqMan OneStep RT-qPCR Kit. Primers used are listed as follows: cyclin D1: Forward: 5'-GGCTCCATGACTGTGGGATC-3', Reverse: 5'-TTCAGCTGCACAGCCCAGAA-3'; Bax: Forward: 5'-GAGGATGATTGCTGATGTG-3', Reverse: 5'-AGTTGAAGTTGCCGTCTG-3'; Bcl-2: Forward: 5' - GGCTACGAGTGGGATACTG-3', Reverse: 5'-GGCTGGAAGGAGAAGATG-3'; Caspase-3: Forward: 5'- GCAGTTTTGTGTGTGTGATT-3', Reverse: 5'- GAGTTTCGGCTTTCCAGT-3'. Reaction mixture was made up according to the protocol, and the reaction program was set as follows: reverse transcription at $45^{\circ} \mathrm{C}$ for 20 min, pre-degeneration at $95^{\circ} \mathrm{C}$ for $5 \mathrm{~min}$, and degeneration at $95^{\circ} \mathrm{C}$ for $15 \mathrm{~s}$ and extend at $60^{\circ} \mathrm{C}$ for $45 \mathrm{~s}$ repeated for 45 cycles. The expression of relative genes was calculated using the $2^{-\Delta \Delta C t}$ method (Livak et al. 2001). GAPDH was used as an internal reference, and the quantification results for each target gene were normalized to GAPDH. Each experiment was repeated for three times.

\section{Western blotting analysis}

Cells in each group were lysed on ice for 30 min using lysis buffer (8 M Urea, $10 \mathrm{mM}$ DTT, $50 \mathrm{mM}$ IAA and protease inhibitor cocktail) after washed with pre-chilled PBS. After lysis, the supernatant was collected after centrifugation, and concentration of proteins were determined using BCA assay. Protein samples $(60 \mu \mathrm{g})$ in four groups were separated with SDS-PAGE electrophoresis. Then proteins were then transferred onto a $0.22-\mu \mathrm{m}$ nitrocellulose membranes using Trans-Blot Turbo system (Bio-Rad). Membranes were firstly incubate with $5 \%$ skim milk, followed with incubation with primary antibodies $(1: 1000)$ at $4^{\circ} \mathrm{C}$ overnight and incubation with secondary antibody (1:5000) for $1 \mathrm{~h}$ at room temperature. Chemiluminiscence was performed using ECL reagents to measure the expression levels of target proteins. The gray values of the bands in each group were quantified using Scion Image software. Gray values were normalized with GAPDH.

\section{Flow cytometry}

Cells in each group were seeded into $100 \mathrm{~mm}$ plate at a concentration of $1 \times 10^{6}$, and after the confluence reached $80-85 \%$, cells were digested with $0.25 \%$ trypsin. After washed with pre-cool PBS, cells were incubated with Annexin V for $15 \mathrm{~min}$ at room temperature, followed with incubation with propidium iodide (PI) for $5 \mathrm{~min}$. The apoptotic cells were detected using FACScan flow cytometry (Becton Dickinson, Heidelberg, Germany).

\section{Enzyme linked immunosorbent assay (ELISA)}

ELISA experiment was proceeded according to the protocol. Briefly, $100 \mu \mathrm{l}$ samples and standards were transferred into each well and incubated for $90 \mathrm{~min}$ at $37^{\circ} \mathrm{C}$, followed with incubation with antibody at $37^{\circ} \mathrm{C}$ for $60 \mathrm{~min}$. After washed with TBS, ABC working solution was added into each well and incubated $37^{\circ} \mathrm{C}$ for $30 \mathrm{~min}$. TMB solution was added after washed with TBS and incubated at $37^{\circ} \mathrm{C}$ for $25 \mathrm{~min}$. The OD value at $450 \mathrm{~nm}$ was measured after TMB stop solution was added using SuPerMax 3100 microplate reader (Shanghai, China). Un-cultured medium was set as a blank control.

\section{Statistical analysis}

Data are presented as the mean $\pm \mathrm{SD}$, each experiment was repeated for three times independently. One-way ANOVA was performed to compare differences between groups followed by Tukey's post-hoc test. $p<0.05$ was set as a statistically significant difference. GraphPad (version 7) was used to analyze the data.

\section{Results}

\section{Effect of cholesterol on proliferation of Ea.hy 926 cells}

In order to detect the effect of cholesterol on proliferation of Ea.hy 926 cells, we performed the MTT assay. The viability rate of Ea.hy 926 cells under $50 \mathrm{mmol} / \mathrm{l}$ cholesterol treatments for 12,24 and $48 \mathrm{~h}$ were $95.3 \pm 4.9 \%, 83.2 \pm 4.1 \%$ and $63.3 \pm$ $3.1 \%$, respectively. The viability was significantly decreased in 24 and $48 \mathrm{~h}$ treatment groups compared with control and $12 \mathrm{~h}$ treatment groups $(p<0.05)$. Thus, we decided to treat endothelial cells for $24 \mathrm{~h}$ to perform the following experiments (Fig. 1A). The viability rate of Ea.hy 926 cells in $\mathrm{CH}$, GO and GD groups under $50 \mathrm{mmol} / \mathrm{l}$ cholesterol stimulation for $24 \mathrm{~h}$ were $87.5 \pm 5.8 \%, 81.5 \pm 5.2 \%$ and $92.6 \pm 6.4 \%$, under $100 \mathrm{mmol} / \mathrm{l}$ cholesterol stimulation for $24 \mathrm{~h}$ were $75.3 \pm 5.1 \%$, $63.6 \pm 4.1 \%$ and $85.3 \pm 5.1 \%$, and under $200 \mathrm{mmol} / \mathrm{l}$ cholesterol stimulation for $24 \mathrm{~h}$ were $62.1 \pm 3.2 \%, 51.4 \pm 3.6 \%$ and 74.5 $\pm 4.8 \%$, respectively (Fig. 1B). Compared with NC group, the viability rate in $\mathrm{GO}$ group was significantly decreased under $50 \mathrm{mmol} / \mathrm{l}$ cholesterol stimulation $(p<0.05)$, significantly decreased in $\mathrm{CH}$ and GD group under $100 \mathrm{mmol} / \mathrm{l}$ cholesterol stimulation $(p<0.05)$ and significantly in $\mathrm{CH}, \mathrm{GO}$ and GD groups under $200 \mathrm{mmol} / \mathrm{l}$ cholesterol stimulation $(p<0.05)$. The viability rate in GO group was significantly decreased under $100 \mathrm{mmol} / \mathrm{l}$ cholesterol stimulation $(p<0.05)$ and 

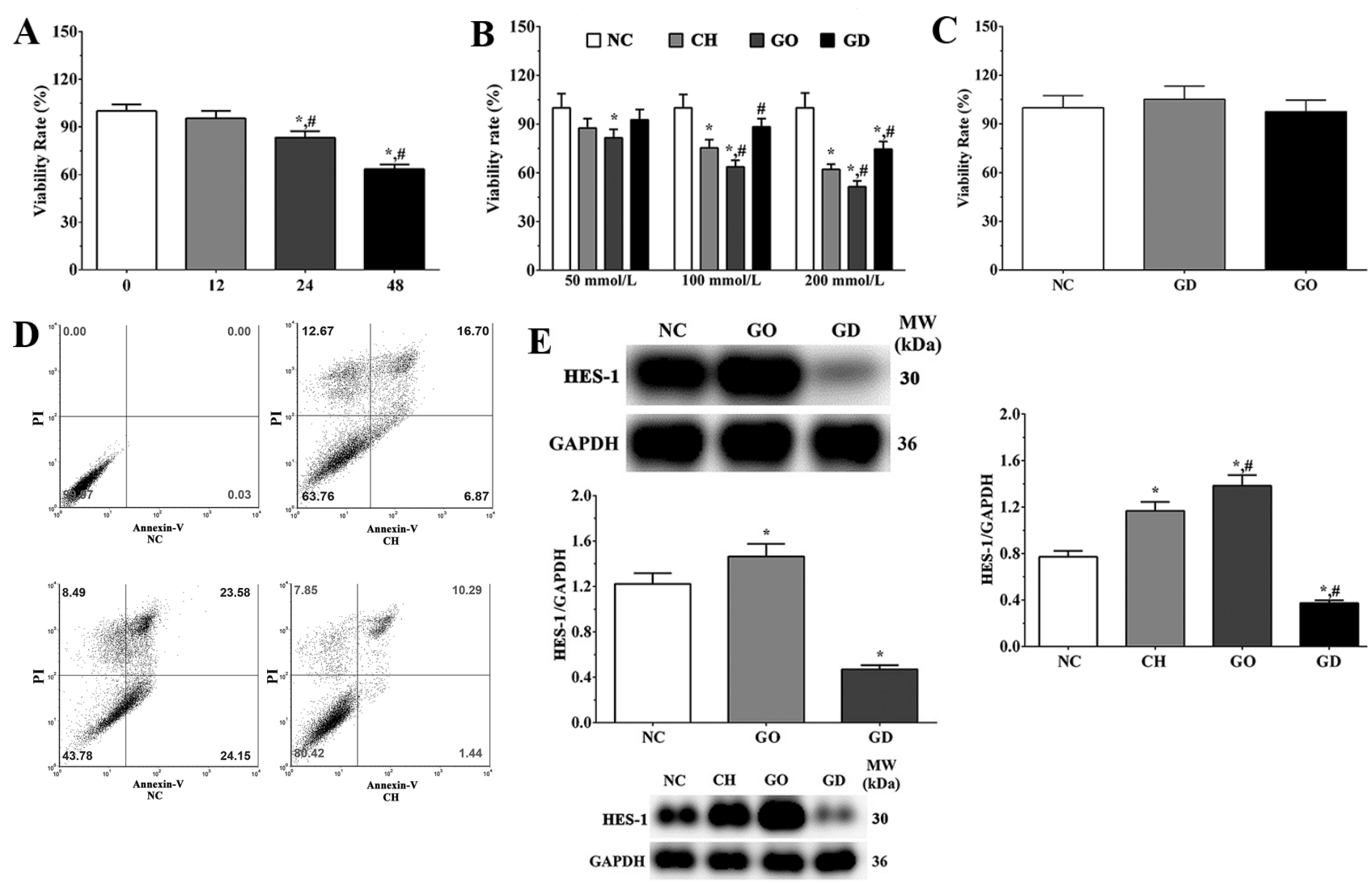

Figure 1. Effect of different cholesterol concentration on proliferation of Ea.hy 926 cells in each group. A. Effect of $50 \mathrm{mmol} / \mathrm{l} \mathrm{cholesterol}$ on proliferation of Ea.hy 926 cells under different treatment period. ${ }^{*} p<0.05 v s .0 \mathrm{~h}$ treatment group, \# $p<0.05 v s .12 \mathrm{~h}$ treatment group. B. Effect of different dose of cholesterol on proliferation of Ea.hy 926 cells for $24 \mathrm{~h}$ treatment. C. Viability rate in each group without cholesterol treatment. D. Effect of cholesterol on apoptosis of Ea.hy 926 cells. E. Expression of HES-1 in each group with and without cholesterol treatment. ${ }^{*} p<0.05 v s$. NC group, $\# p<0.05 v s$. CH group. Data was presented as mean \pm SD, each experiment was repeated for three times independently. NC, control group; $\mathrm{CH}$, cholesterol stimulation group; GO, HES-1 overexpression with cholesterol stimulation group; GD, HES-1 knockdown with cholesterol stimulation group; PI, propidium iodide.

significantly decreased in GO and GD group and significantly increased in GD groupunder $200 \mathrm{mmol} / \mathrm{l}$ cholesterol stimulation $(p<0.05)$ compared with $\mathrm{CH}$ group. Thus, we used 100 $\mathrm{mmol} / \mathrm{l}$ cholesterol treated for $24 \mathrm{~h}$ to perform the following experiment. And the viability rate in NC, GD and go group was $100.0 \pm 7.4,105.2 \pm 8.1$ and $97.6 \pm 7.1$, and the viability rate was not significantly changed in these groups (Fig. 1C). The expression of HES-1 in NC, GO and GD groups without cholesterol treatment were $1.22 \pm 0.09,1.46 \pm 0.11$ and $0.47 \pm$ 0.04 , respectively. The expression of HES- 1 was significantly increased in GO group $(p<0.05)$ and significantly decreased in GD group $(p<0.05)$ compared with NC group, indicating the successfully establish of the cell model. The expressions of HES- 1 were $0.77 \pm 0.05,1.17 \pm 0.08,1.38 \pm 0.09$ and 0.37 \pm 0.02 in $\mathrm{NC}, \mathrm{CH}, \mathrm{GO}$ and GD group, respectively. The expression level of HES-1 was significantly up-regulated in $\mathrm{CH}$ and GO group compared with NC group $(p<0.05)$, and was significantly down-regulated in GD group $(p<0.05)$.
The expression level of HES-1 was significantly up-regulated in GO group compared with $\mathrm{CH}$ group $(p<0.05)$ and was significantly down-regulated in GD group ( $p<0.05$, Fig. 1E). And the percentage of apoptotic cells in each group were 0.03 $\pm 0.01 \%, 36.24 \pm 3.21 \%, 56.22 \pm 5.48 \%$ and $19.58 \pm 2.03 \%$ (Fig. 1D). Compared with NC group, the apoptic cells were significantly increased in all treatment groups $(p<0.05)$, and was significantly increased in GO group vs. $\mathrm{CH}$ group $(p<$ 0.05 ). These results indicating that cholesterol treatment could significantly activate the apoptosis process in cells, and knockdown of HES-1 could reverse this change into a normal level.

\section{Altering in expression of apoptosis related genes under cholesterol stimulation}

Cytokines including TNF- $\alpha$, would activate downstream molecular, such as NF- $\kappa \mathrm{B}$, further affects multiple downstream proteins, such as BAX, Bcl-2 and caspase cascades. As shown 

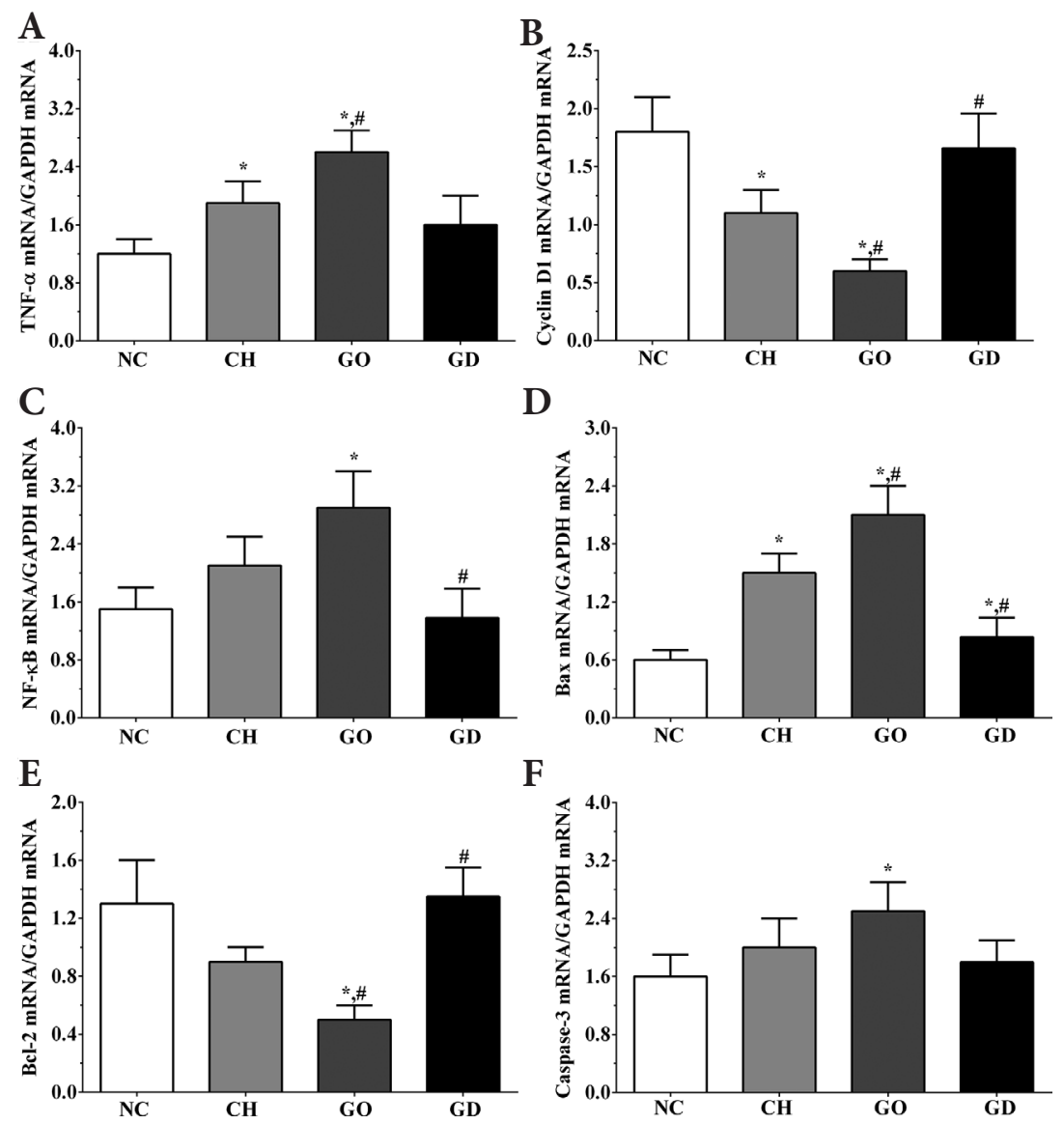

Figure 2. Effect of cholesterol on gene expression of apoptosis related molecules TNF- $\alpha$

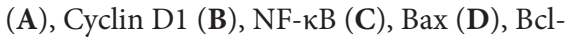
2 (E) and caspase-3 (F) of Ea.hy 926 cells in each group. GAPDH was used as an internal control. ${ }^{*} p<0.05 v s$. NC group, $\# p<0.05$ vs. $\mathrm{CH}$ group. Data was presented as mean $\pm \mathrm{SD}$, each experiment was repeated for three times independently. For abbreviations, see Fig. 1.

in Fig. 2, the expression level of TNF- $\alpha$ in NC, CH, GO and GD groups were $1.1 \pm 0.2,1.8 \pm 0.3,2.6 \pm 0.5$ and $1.6 \pm 0.5$, respectively. The expression of TNF- $\alpha$ were significantly upregulated in $\mathrm{CH}$ and $\mathrm{GO}$ groups $(p<0.05) v s$. NC group, and compared with $\mathrm{CH}$ group, the expression of TNF- $\alpha$ was significantly up-regulated in GO group $(p<0.05)$. The expression level of cyclin D1 in these groups were $1.8 \pm 0.4$, $1.1 \pm 0.2,0.6 \pm 0.1$ and $1.7 \pm 0.3$. Compared with NC group, the expression of cyclin D1 was significantly decreased in $\mathrm{CH}$ and GO groups $(p<0.05)$, and compared with $\mathrm{CH}$ group, the expression of cyclin D1 was significantly decreased in GO group $(p<0.05)$ and significantly increased in GD group $(p<$ $0.05)$. The expression level of NF- $\kappa B$ in these groups were 1.5 $\pm 0.3,2.1 \pm 0.4,2.8 \pm 0.5$ and $1.3 \pm 0.4$. Compared with NC group, the expression of NF- $\kappa \mathrm{B}$ was significantly up-regulated in $\mathrm{GO}$ group $(p<0.05)$. When compared with $\mathrm{CH}$ group, the expression of NF- $\kappa \mathrm{B}$ was significantly down-regulated in GD group. The expression level of Bax in these groups were $0.6 \pm$ $0.1,1.4 \pm 0.3,2.2 \pm 0.4$ and $0.9 \pm 0.2$. Compared with NC group, the expression of Bax was significantly up-regulated in all cholesterol treatment groups $(p<0.05)$, and was significantly up-regulated in GO group and significantly down-regulated in GD group vs. $\mathrm{CH}$ group $(p<0.05)$. The expression level of
$\mathrm{Bcl}-2$ in these groups were $1.3 \pm 0.3,0.9 \pm 0.1,0.5 \pm 0.1$ and $1.4 \pm 0.2$. Compared with $\mathrm{NC}$ and $\mathrm{CH}$ group, the expression of $\mathrm{Bcl}-2$ was significantly down-regulated in GO group $(p<0.05)$, and the expression of Bcl-2 was significantly up-regulated in GD group $(p<0.05) v s$. CH group. The expression level of caspase- 3 in these groups were $1.6 \pm 0.3,2.0 \pm 0.4,2.4 \pm 0.4$ and $1.8 \pm 0.2$. The expression of caspase- 3 was significantly up-regulated in GO group vs. NC group $(p<0.05)$.

\section{Expression levels of apoptosis related molecules under cholesterol stimulation}

The expression of Bax were $0.33 \pm 0.02,0.83 \pm 0.06,1.11 \pm 0.07$ and $0.56 \pm 0.04$ in $\mathrm{NC}, \mathrm{CH}, \mathrm{GO}$ and GD group, respectively (Fig. 3). The expression of BAX were significantly up-regulated in all treatment groups $(p<0.05)$ compared with NC group, and the expression of BAX was significantly up-regulated in GO group $(p<0.05)$ and significantly down-regulated in GD group $(p<0.05)$ vs. $\mathrm{CH}$ group. The expression of Bcl-2 were $1.02 \pm 0.07,0.88 \pm 0.06,0.68 \pm 0.05$ and $0.73 \pm 0.05$ in these groups. Compared with NC group, the expression of $\mathrm{Bcl}-2$ were significantly down-regulated in all treatment groups $(p<$ 0.05), and compared with $\mathrm{CH}$ group, the expression of Bcl-2 
were significantly down-regulated in GO and GD group $(p<$ $0.05)$. The expression of caspase- 3 were $0.44 \pm 0.03,0.88 \pm 0.06$, $0.91 \pm 0.06$ and $0.30 \pm 0.02$ in these groups. The expression of caspase-3 were significantly up-regulated in $\mathrm{CH}$ and $\mathrm{GO}$ groups $(p<0.05)$ and significantly decreased in GD group $(p<$ $0.05) v s$. NC group, and the expression of $\mathrm{Bcl}-2$ was significantly decreased in GD group $v s$. $\mathrm{CH}$ group $(p<0.05)$. The expression of caspase- 9 were $0.12 \pm 0.01,0.68 \pm 0.05,0.96 \pm 0.06$ and $0.51 \pm 0.03$ in these groups. The expression of caspase- 9 were significantly up-regulated in all treatment groups compared with NC group $(p<0.05)$, and was significantly up-regulated in GO group $(p<0.05)$ and significantly down-regulated in GD group $(p<0.05) v s$. $\mathrm{CH}$ group. BIM is a downstream molecular of PI3K/AKT signaling pathway, which performs an improtant role in apoptosis process via regulation of Bcl-2/ $\mathrm{BAX}$. And the ratio of $\mathrm{p}-\mathrm{BIM} / \mathrm{BIM}$ were $0.76 \pm 0.05,1.52 \pm$ $0.10,1.51 \pm 0.10$ and $0.87 \pm 0.06$ in these groups. Compared with NC group, the ratio of $\mathrm{p}$-BIM/BIM were significantly up-regulated in $\mathrm{CH}$ and $\mathrm{GO}$ groups $(p<0.05)$, and the ratio of p-BIM/BIM was significantly down-regulated in GD group compared with $\mathrm{CH}$ group $(p<0.05)$. Combined with the results of qPCR, the cellular apoptosis process was activated after cholesterol treatment, and knockdown of HES-1 would reverse these changes, presented a protective role.
Effect of cholesterol on secretion of angiogenesis related molecules

Maintainence of harmonious status of endothelial cells relys on the secertion of multiple factors in circulation blood, thus, angiogenesis process was critical for the proliferation of endothelial cells. As shown in Fig. 4, the concentration of TGF- $\beta$ in cultured medium were 531.2 $\pm 23.1,384.6 \pm 18.4$, $265.1 \pm 12.2$ and $486.2 \pm 21.0$ in NC, CH, GO and GD groups, respectively. Compared with $\mathrm{NC}$ group, the concentration of TGF- $\beta$ was significantly down-regulated in $\mathrm{CH}$ and GO groups $(p<0.05)$, and was significantly down-regulated in GO group $(p<0.05)$, significantly up-regulated in GD group $(p<0.05) v s$. $\mathrm{CH}$ group. The concentrations of IGF in cultured medium were 1321.2 $\pm 41.1,984.6 \pm 31.2,841.2$ \pm 26.5 and $1287.1 \pm 29.3$ in these groups. The concentration of IGF was significantly down-regulated in $\mathrm{CH}$ and GO group compared with NC group $(p<0.05)$, significantly down-regulated in GO group $(p<0.05)$ and significantly up-regulated in GD group $(p<0.05)$ vs. $\mathrm{CH}$ group. The concentration of VEGF- $\alpha$ in cultured medium were $154.2 \pm$ $10.1,105.6 \pm 9.2,83.2 \pm 5.5$ and $134.1 \pm 9.5$ in these groups. Compared with NC group, the concentration of VEGF- $\alpha$ was significantly down-regulated in $\mathrm{CH}$ and GO group $(p<$
A

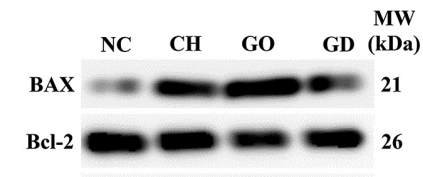

Cleavge caspase-3 35

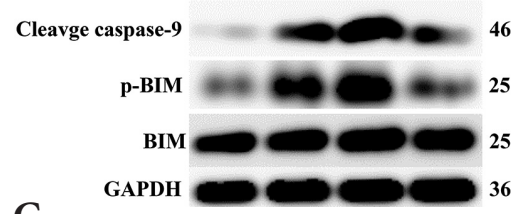

C

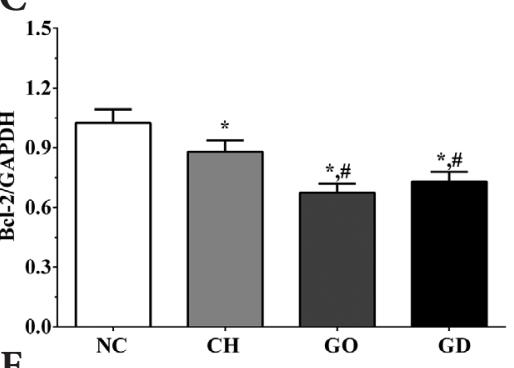

E

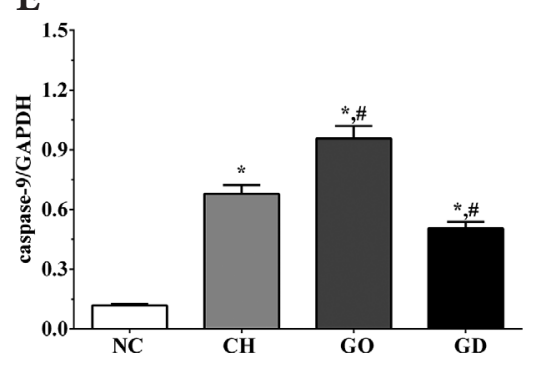

B

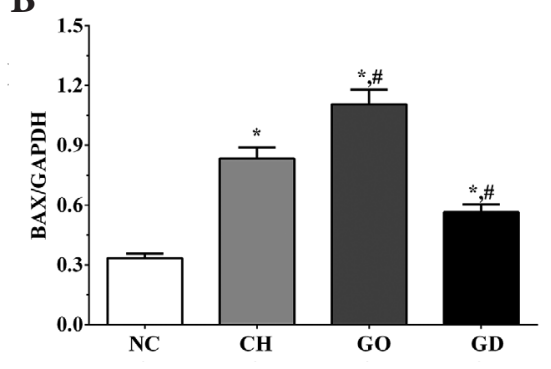

D
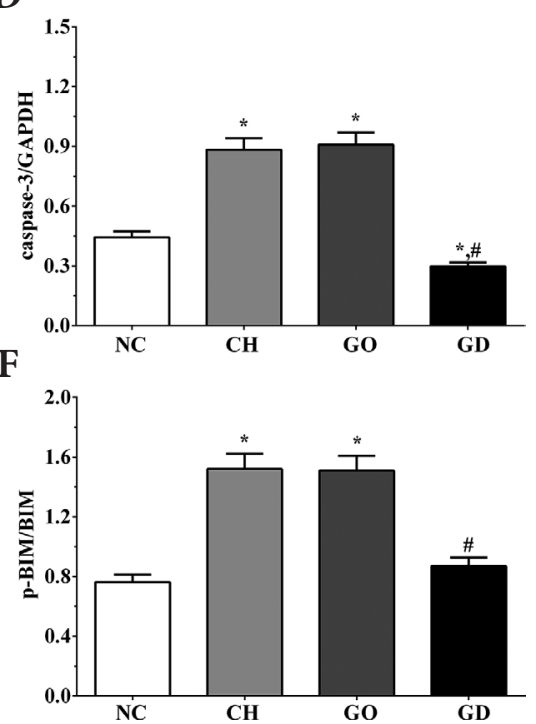

Figure 3. Effect of cholesterol on expression of apoptosis related proteins of Ea.hy 926 cells in each group. A. Western blotting analysis of Bax, Bcl-2, cleavge caspase-3, cleavge caspase-9, p-BIM and BIM. Quantitative analysis of Bax (B), Bcl-2 (C), cleavge caspase-3 (D), cleavge caspase-9 (E) and p-BIM/BIM (F). GAPDH was used as an internal control. ${ }^{*} p<0.05$ vs. NC group, \# $p<0.05 v s$. $\mathrm{CH}$ group. Data was presented as mean $\pm \mathrm{SD}$, each experiment was repeated for three times independently. For abbreviations, see Fig. 1. 
$0.05)$, and significantly down-regulated in GO group $v s . \mathrm{CH}$ group $(p<0.05)$. The concentrations of PDGF in cultured medium were $3520.2 \pm 60.5,2965.1 \pm 41.2,2136.5 \pm 35.0$ and $3294.1 \pm 37.3$ in these groups. Compared with NC group, concentration of PDGF was significantly down-regulated in cholesterol treatment groups $(p<0.05)$, and compared with $\mathrm{CH}$ group, concentration of PDGF was significantly down-regulated in GO group $(p<0.05)$ and significantly up-regulated in GD group $(p<0.05)$. These results indicate that knockdown of HES-1 expression might promote the proliferation via promoting angiogenesis process.

Effect of cholesterol on expression levels of Wnt/PI3K/AKT/ $\beta$-catenin signaling pathway

As shown in Fig. 5, the expression of Wnt were $1.11 \pm 0.07$, $1.14 \pm 0.08,0.55 \pm 0.04$ and $0.75 \pm 0.05$ in these groups. The expression level of Wnt was significantly down-regulated in GO and GD group $(p<0.05)$ compared with $\mathrm{NC}$ and $\mathrm{CH}$ group. The ratio of $\mathrm{p}-\mathrm{AKT} / \mathrm{AKT}$ were $1.19 \pm 0.08,0.98 \pm 0.07$, $0.63 \pm 0.04$ and $1.43 \pm 0.10$ in these groups. The ratio of $\mathrm{p}$ AKT/AKT was significantly down-regulated in all treatment groups $(p<0.05) v s$. NC group, significantly down-regulated in GO group and significantly up-regulated in GD group $v s$. $\mathrm{CH}$ group $(p<0.05)$. The expression of $\beta$-catenin was $1.06 \pm$ $0.07,1.06 \pm 0.07,0.63 \pm 0.04$ and $0.76 \pm 0.05$ in these groups. The expression of $\beta$-catenin was significantly down-regulated in GO and GD groups $(p<0.05)$ compared with $\mathrm{NC}$ and $\mathrm{CH}$ group. And the expression of $\mathrm{c}-\mathrm{Myc}$ were $0.73 \pm 0.05$, $0.36 \pm 0.02,0.19 \pm 0.01$ and $0.51 \pm 0.03$ in these groups. The expression of $\mathrm{c}-\mathrm{Myc}$ was significantly in all treatment groups compared with NC group $(p<0.05)$, significantly down-regulated in GO group $(p<0.05)$ and significantly up-regulated in GD group vs. CH group.

\section{Discussion}

Atherosclerosis is a basic pathology process of cardiovascular disease (CVD), leading to the occurrence of myocardial infarction, stroke and peripheral artery disease (Mozaffarian et al. 2016). Formation of atherosclerotic lesions is the main characteristic of atherosclerosis which is induced by excessive deposition of cholesterol in arterial intima. Research on clinical trials and animal models have found the direct correlation between concentration of cholesterol in plasma and the incidence of CVD (Barton et al. 2013). Meanwhile, cholesterol is critical for cellular biology and maintenance of cholesterol in mammals is important for systemic mechanisms. HES-1 plays an important role in cell proliferation and differentiation processes of multiple cell types, and also related to development of aorta and ventricular septal defects as well as CVD. In present study, we constructed a cell model in Ea.hy 926 cells of HES-1 overexpression and knockdown model, observed the effect of cholesterol on growth of Ea.hy 926 cells and explored possible mechanism. We found that the activation of Wnt/ $\beta$-catenin/c-Myc signaling pathway after HES-1 overexpressed, while the activity of PI3K/AKT signaling pathway was inhibited. Besides, we also found that the cellular apoptosis process was activated
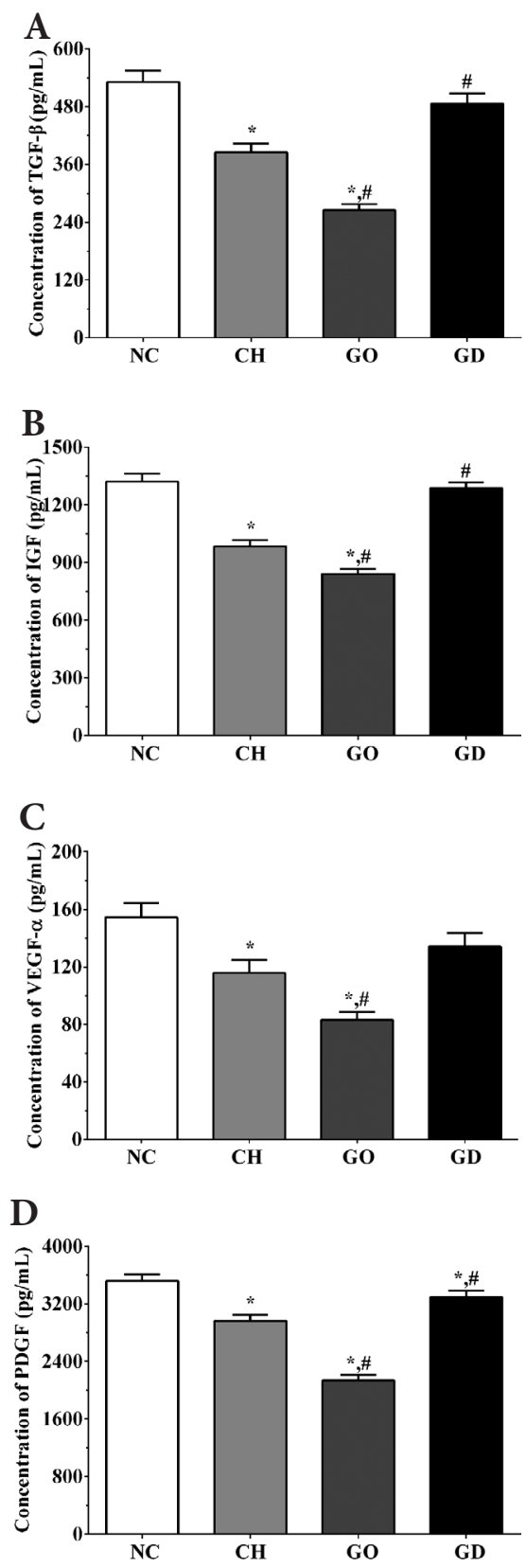

Figure 4. Effect of cholesterol on secretion of angiogenesis related molecules TGF- $\beta$ (A), IGF (B), VEGF- $\alpha(\mathbf{C})$ and PDGF (D) in cultured medium. ${ }^{*} p<0.05 v$ s. NC group, $\# p<0.05 v s$. $\mathrm{CH}$ group. Data was presented as mean $\pm S D$, each experiment was repeated for three times independently. For abbreviations, see Fig. 1. 
after cholesterol treatment, and this effect was enlarged after HES-1 overexpressed. The concentration of key cytokines associated with angiogenesis was also down-regulated after cholesterol stimulation and HES-1 knockdown would reverse the phenomena, thus we speculated that HES-1 might be a new threptic target.

Wnt signaling pathway is a critical pathway not only in development biology, but also in vasculogenesis (Clevers et al. 2012). Vasculogenesis is the initial step of vasculature formation, which needs differentiations of endothelial cells from mesodermal precursors. And this step is regulated by multiple pathways, including Wnt signaling pathway. Previous study found that the differentiation process of embryonic stem cells into endothelial cells was under the regulation of Wnt/ $\beta$-catenin signaling pathway (Yang et al. 2009). Besides, R-spondin-3 also contributes to vasculogenesis and angiogenesis. Previous study found the role of R-spondin-3 in Xenopus and mice embryos could promote the differentiation process of endothelial cells toward angioblasts and activation of $\beta$-catenin pathway, leading to the increasing in concentration of vascular endothelial growth factor (VEGF) (Kazanskaya et al. 2008). In Wnt signaling pathway, phosphorylation process of $\beta$-catenin was commonly undergo via suppression of CK1 and GSK-3 which allows formation of complex of $\beta$-catenin with various transcription factors including VEGF factor, c-Myc and c-Jun, further inducing the increased expression of target genes (Clevers et al. 2012). However, we noticed that the expression of Wnt and $\beta$-catenin was not significantly changed after inhibition and overexpression of HES-1, indicating that Wnt/ $\beta$-catenin signaling pathway was not significantly affect after inhibition of HES-1 expression under cholesterol stimulation. Expression of c-Myc is commonly seen in endothelin (ET)stimulated vascular smooth muscle cells (VSMC) (Chen et al. 2006). Previous study found that increased expression of ET in experimental model of atherosclerosis was associated with endothelial dysfunction and neovascularization, which is critical for initiation of atherosclerosis (de Nigris et al. 2003). Changing in expression of c-Myc would induce the oxidative stress and DNA damage in cells, besides, previous studies found that c-Myc participates in early atherogenesis in vitro and in vivo experiments (de Nigris et al. 2001; Cárcamo et al. 2006). These effects were also regulated by AKT, p38 mitogen-activated protein kinase (MAPK) and Ras signaling pathways, which are critical for cellular survival, proliferation and apoptosis (Griendling et al. 2000). The expression of c-Myc was significantly increased after inhibition of AKT, a critical number of PI3K/PTEN/AKT/ mTORC1 signaling pathway (Toker et al. 2014). Previous study also found that activation of PI3K/AKT would increase the expression of c-Myc while inhibition of PI3K/AKT would reduce the expression of c-Myc (Kumar et al. 2016). PI3K/ AKT signaling pathway performs an important role in cellular survival, endothelial cell migration and angiogenesis (Huang et al. 2016). Activation of phosphatase and tensin homolog protein (PTEN) could reduce the activation of PI3K/AKT signaling pathway, dysfunction of PTEN was commonly seen in cancers for it could promote migration
A

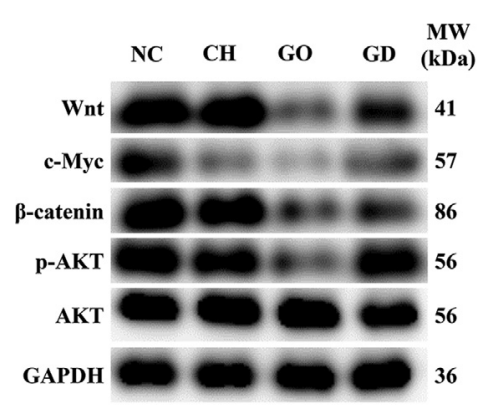

B
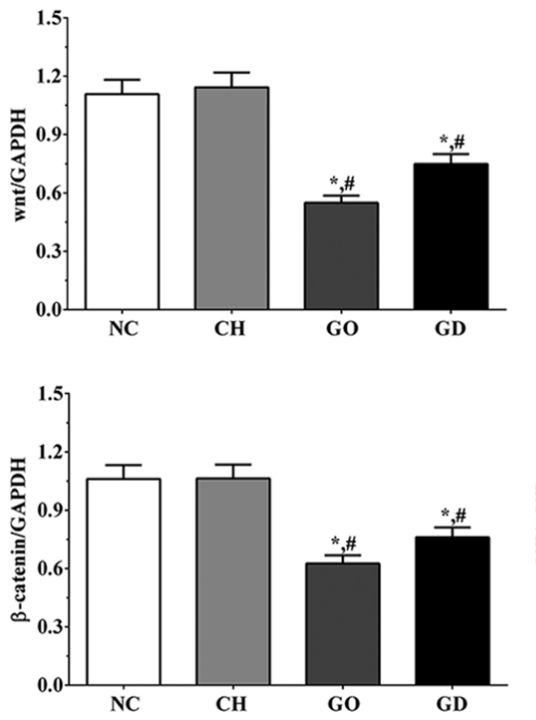
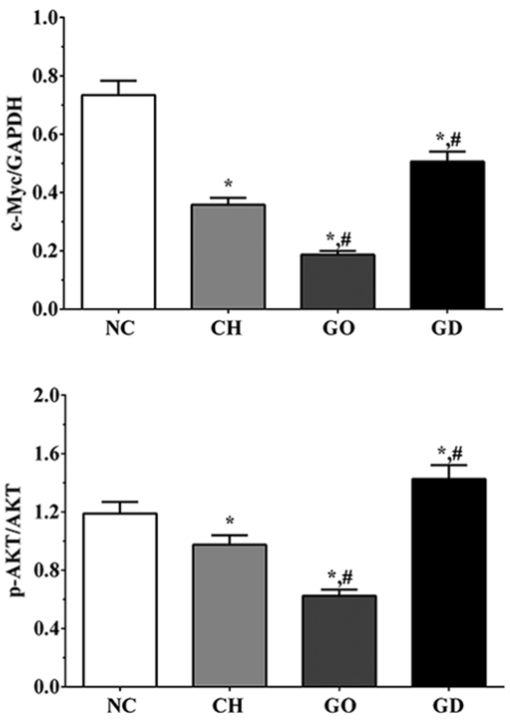

Figure 5. Effect of cholesterol on activation of Wnt/PI3K/AKT/ $\beta$-catenin signaling pathway. A. Western blotting analysis of HES-1, Wnt, c-Myc, $\beta$-catenin, p-AKT and AKT. B. Quantitative analysis of Wnt, c-Myc, $\beta$-catenin and p-AKT/AKT. GAPDH was used as an internal control. ${ }^{*} p<0.05 v s$. NC group, $\# p<0.05 v s$. CH group. Data was presented as mean $\pm \mathrm{SD}$, each experiment was repeated for three times independently. For abbreviations, see Fig. 1. 
and survival of cancer cells. This effect was indirect mediated by induced the activation of mammalian target of rapamycin (mTOR) activity by PTEN (Mao et al. 2012). HES-1 is a target gene of Notch signaling pathway, the expression of HES-1 would be increased at transcription level with the activation of Notch pathway, which would result in reduction of PTEN activation. Accumulation of PIP3 leads to the phosphorylation of AKT kinase at site of Ser473 or Thr 308, followed with phosphorylated at Ser473 by mTORC2 resulting in fully activation of AKT (Hubbard et al. 2014). Activation of Notch leads to the expression of HES-1, resulting in reduction of PTEN expression. These processes would activate $\mathrm{PI} 3 \mathrm{~K} / \mathrm{AKT} / \mathrm{mTOR}$ signaling pathway, promoting cellular proliferation, survival and angiogenesis (Maccario et al. 2007; Palomero et al. 2008).

TNF- $\alpha$ is an important cytokine in regulation of inflammation and cytokine network, maintaining its homeostasis (Balkwill et al. 2009), and was found participated in hemorrhagic necrosis process of tumor cells in both human and mice (Carswell et al. 1975). The releasing of TNF-a would also lead to the occurrence of inflammatory bowel disease, psoriasis and rheumatoid arthritis (Ma et al. 2016). Inflammatory diseases inducing by the release of TNF- $\alpha$ were not associated with its ability to induce NF- $\kappa \mathrm{B}$ and MAPKdependent signaling pathway, but for its ability to induce cell death. Previous study found that the inflammatory cell death in mice model induced by TNF- $\alpha$ could be reduced by genetic deletion of cell death components, including cytokines, chemokines and DMAPs (Rickard et al. 2014). Bcl-2 protein family contains plenty numbers, including prosurvival proteins (Bcl-2, Bcl-B, Mcl-1, Bfl-1/A1, Bcl-w and $\mathrm{Bcl}-\mathrm{xl}$ ), pro-apoptotic $\mathrm{BH} 3$-only proteins, and pro-apoptotic effectors (BAK and BAX) (Ke et al. 2018). Cytotoxic stimuli including DNA damage, chemotherapeutic agents and cytokines would induce down-regulation of the expression of pro-survival $\mathrm{Bcl}-2$ protein and the up-regulation in $\mathrm{BH} 3$ only proteins expression (Czabotar et al. 2014). BAD specific bind with $\mathrm{Bcl}-\mathrm{w}, \mathrm{Bcl}-\mathrm{xl}$ and $\mathrm{Bcl}-2$, while NOXA selectively inhibits Bfl-1 and Mcl-1. BID, BIM and PUMA could bind with all pro-survival proteins. Followed with pro-survival proteins sequestered by $\mathrm{BH} 3$-only proteins, BAX and BAK could disrupt the outer membrane of mitochondrial, leading to the release of cytochrome $c$, activation of caspase cascade (including caspase-3) and finally cause the execution of apoptosis (Wong et al. 2011). Besides, some $\mathrm{BH} 3$-only proteins, including BIM, PUMA and BID, could directly bind with BAX or BAK, leading to the conformational changes and activation of BAX/BAK (Czabotar et al. 2013).

Previous study found that angiogenesis function of PI3K/AKT signaling pathway was mediated by increasing the expression of VEGF factor further increase the activity of endothelial nitric oxide synthase in endothelial cells (Manning et al. 2007). Besides, activation of Wnt/ $\beta$-catenin signaling pathway could directly stimulate the expression of EGF and its downstream pathway, including PI3K/AKT/ mTOR signaling pathway (Popescu et al. 2016). Secretion of platelet-derived growth factor (PDGF) could induce the aggregation of pericytes at the site of newly formed vessels, as well as promoting the formation of basement membrane via ANG/TIE pathway, a downstream pathway of Wnt/ $\beta$-catenin (Ferrara et al. 2005). Previous study has found that TGF- $\beta$ could enhance the process of angiogenesis via increasing the function of endothelial cells and the expression of VEGF (Pertovaara et al. 1994). Increasing expression of VEGF could promote angiogenesis through recruitment of endothelial cells (Petersen et al. 2010). Effect of TGF- $\beta$ on endothelial cells could be contradictory through relationship between TGF- $\beta$ and ALK1 and ALK5 receptors. Low concentration of TGF- $\beta$ could interact with ALK1 receptors and increase the expression of metalloproteases-2 (MMP-2) and MMP-9, further activate endothelial cells. While higher concentration of TGF- $\beta$ would interact with ALK 5 receptor, further hinder angiogenesis (Goumans et al. 2002).

In this study, we firstly established HES-1 overexpression and knockdown cell model in Ea.hy 926 cells and observe the effect of HES-1 on proliferation of Ea.hy 926 cells under cholesterol stimulation. Then we found the down-regulated expression of pro-apoptosis factors and increased expression of anti-apoptosis factors in Ea.hy 926 cells at protein and gene levels. We further found that the activity of Wnt/ $\beta$-catenin/AKT signaling pathway was inhibited after cholesterol stimulation and the effect was enlarged after HES-1 overexpression, while alleviated after knockdown of HES-1. Besides, we found that the concentration of angiogenesis factors in cultured medium was also down-regulated after cholesterol stimulation, and was increased after the expression of HES-1 inhibited. Inhibition of activity of $\mathrm{Wnt} / \beta$-catenin leads to the inhibition of the activity of PI3K/AKT/c-Myc signaling pathway, resulting in activition of angiogenesis process and inhibition of cellular apoptosis process, further promotes the proliferation of cells. Thus, we speculated that HES-1 might be a new therapeutic strategy for cardiovascular disease. However, more experiments on endothelial cells and animal models would be helpful for us to explore the mechanism of cholesterol and cardiovascular diseases, and human trial was also critical in our following experiments.

Acknowledgements. This work was supported by Tianjin science and technology project (16ZXHLSY00120).

Conflict of interest. The author declared no conflict of interest.

Author Contributions. Yun Shi, Ya Su and Chuanli Liu performed the experiment, Jinglong Tao analyzed the data, Hao $\mathrm{Fu}$ and Chengzhi Lu designed the experiment and revised the manuscript. 


\section{References}

Abderrahmani A, Niederhauser G, Lenain V, Regazzi R, Waeber G (2005): The hairy and enhancer of split 1 is a negative regulator of the repressor element silencer transcription factor. FEBS Lett. 579, 6199-6204 https://doi.org/10.1016/j.febslet.2005.09.093

Balkwill F (2009): Tumour necrosis factor and cancer. Nat. Rev. Cancer 9, 361-371 https://doi.org/10.1038/nrc2628

Barton M (2013): Cholesterol and atherosclerosis, modulation by oestrogen. Curr. Opin. Lipidol. 24, 214-220 https://doi.org/10.1097/MOL.0b013e3283613a94

Canver MC, Haeussler M, Bauer DE, Orkin SH, Sanjana NE, Shalem O, Yuan GC, Zhang F, Concordet JP, Pinello L (2018): Integrated design, execution, and analysis of arrayed and pooled CRISPR genome-editing experiments. Nat. Protoc. 13, 946-986 https://doi.org/10.1038/nprot.2018.005

Cárcamo JM, Golde DW (2006): Antioxidants prevent oxidative DNA damage and cellular transformation elicited by the overexpression of c-MYC. Mutat. Res. 593, 64-79 https://doi.org/10.1016/j.mrfmmm.2005.06.015

Carswell EA, Old LJ, Kassel RL, Green S, Fiore N, Williamson B (1975): An endotoxin-induced serum factor that causes necrosis of tumors. Proc. Natl. Acad. Sci. U.S.A. 72, 3666-3670 https://doi.org/10.1073/pnas.72.9.3666

Chen S, Qiong Y, Gardner DG (2006): A role for p38 mitogenactivated protein kinase and c-myc in endothelin-dependent rat aortic smooth muscle cell proliferation. Hypertension 47, $252-258$

https://doi.org/10.1161/01.HYP.0000198424.93598.6b

Clevers H, Nusse R (2012): Wnt/ $\beta$-catenin signaling and disease. Cell 149, 1192-1205 https://doi.org/10.1016/j.cell.2012.05.012

Czabotar PE, Lessene G, Strasser A, Adams JM (2014): Control of apoptosis by the BCL-2 protein family, implications for physiology and therapy. Nat. Rev. Mol. Cell. Biol. 15, 49-63 https://doi.org/10.1038/nrm3722

Czabotar PE, Westphal D, Dewson G, Ma S, Hockings C, Fairlie WD, Lee EF, Yao S, Robin AY, Smith BJ, ey al. (2013): Bax crystal structures reveal how $\mathrm{BH} 3$ domains activate Bax and nucleate its oligomerization to induce apoptosis. Cell 152, 519-531 https://doi.org/10.1016/j.cell.2012.12.031

de Nigris F, Lerman LO, Rodriguez-Porcel M, De Montis MP, Lerman A, Napoli C (2001): c-myc activation in early coronary lesions in experimental hypercholesterolemia. Biochem. Biophys. Res. Commun. 281, 945-950 https://doi.org/10.1006/bbrc.2001.4431

de Nigris F, Sica V, Herrmann J, Condorelli G, Chade AR, Tajana G, Lerman A, Lerman LO, Napoli C (2003): c-Myc oncoprotein, cell cycle-related events and new therapeutic challenges in cancer and cardiovascular diseases. Cell Cycle 2, 325-328 https://doi.org/10.4161/cc.2.4.414

Ference BA, Ginsberg HN, Graham I, Ray KK, Packard CJ, Bruckert E, Hegele RA, Krauss RM, Raal FJ, Schunkert H, et al. (2017): Low-density lipoproteins cause atherosclerotic cardiovascular disease. 1. Evidence from genetic, epidemio- logic, and clinical studies. A consensus statement from the European Atherosclerosis Society Consensus Panel. Eur. Heart J. 38, 2459-2472

https://doi.org/10.1093/eurheartj/ehx144

Ferrara N, Kerbel RS (2005): Angiogenesis as a therapeutic target. Nature 438, 967-974

https://doi.org/10.1038/nature04483

Goldstein JL, Brown MS (2015): A century of cholesterol and coronaries, from plaques to genes to statins. Cell 161, 161-172 https://doi.org/10.1016/j.cell.2015.01.036

Goumans MJ, Valdimarsdottir G, Itoh S, Rosendahl A, Sideras P, ten Dijke P (2002): Balancing the activation state of the endothelium via two distinct TGF-beta type I receptors. EMBO J. 21, 1743-1753 https://doi.org/10.1093/emboj/21.7.1743

Griendling KK, Sorescu D, Lassègue B, Ushio-Fukai M (2000): Modulation of protein kinase activity and gene expression by reactive oxygen species and their role in vascular physiology and pathophysiology. Arterioscler. Thromb. Vasc. Biol. 20, 2175-2183 https://doi.org/10.1161/01.ATV.20.10.2175

Huang C, Lin MZ, Cheng D, Braet F, Pollock CA, Chen XM (2016): Kca3.1 mediates dysfunction of tubular autophagy in diabetic kidneys via pi3k/akt/mtor signaling pathways. Sci. Rep. 6, 23884 https://doi.org/10.1038/srep23884

Hubbard PA, Moody CL, Murali R (2014): Allosteric modulation of Ras and the PI3K/AKT/mTOR pathway, emerging therapeutic opportunities. Front. Physiol. 5, 478 https://doi.org/10.3389/fphys.2014.00478

Ishibashi M, Ang SL, Shiota K, Nakanishi S, Kageyama R, Guillemot F (1995): Targeted disruption of mammalian hairy and Enhancer of split homolog-1 (HES-1) leads to up-regulation of neural helix-loop-helix factors, premature neurogenesis, and severe neural tube defects. Genes. Dev. 9, 313-3148 https://doi.org/10.1101/gad.9.24.3136

Kageyama R, Ohtsuka T, Hatakeyama J, Ohsawa R (2005): Roles of bHLH genes in neural stem cell differentiation. Exp. Cell. Res. 306, 343-348 https://doi.org/10.1016/j.yexcr.2005.03.015

Kazanskaya O, Ohkawara B, Hatakeyama J, Ohsawa R (2008): The Wnt signaling regulator R-spondin 3 promotes angioblast and vascular development. Development 135, 3655-3664 https://doi.org/10.1242/dev.027284

Ke FFS, Vanyai HK, Cowan AD, Delbridge ARD, Whitehead L, Grabow S, Czabotar PE, Voss AK, Strasser A (2018): Embryogenesis and adult life in the absence of intrinsic apoptosis effectors BAX, BAK, and BOK. Cell 173, 1217-1230 https://doi.org/10.1016/j.cell.2018.04.036

Kumar A, Marqués M, Carrera AC (2016): Phosphoinositide 3-kinase activation in late $\mathrm{G} 1$ is required for c-Myc stabilization and S phase entry. Mol. Cell. Biol. 26, 9116-9125 https://doi.org/10.1128/MCB.00783-06

Livak KJ, Schmittgen TD (2001): Analysis of relative gene expression data using real-time quantitative PCR and the 2(-Delta Delta C(T)) Method. Methods 25, 402-408 https://doi.org/10.1006/meth.2001.1262

Ma K, Zhang H, Baloch Z (2016): Pathogenetic and therapeutic applications of tumor necrosis factor- $\alpha$ (TNF- $\alpha$ ) in major 
depressive disorder. A Systematic Review. Int. J. Mol. Sci. 17, E733 https://doi.org/10.3390/ijms17050733

Maccario H, Perera NM, Davidson L, Downes CP, Leslie NR (2007) PTEN is destabilized by phosphorylation on Thr366. Biochem. J. 405, 439-444 https://doi.org/10.1042/BJ20061837

Majidinia M, Alizadeh E, Yousefi B, Akbarzadeh M, Mihanfar A, Rahmati-Yamchi M, Zarghami N (2017): Co-inhibition of Notch and NF- $\mathrm{KB}$ signaling pathway decreases proliferation through downregulating IкB- $\alpha$ and Hes- 1 expression in human ovarian cancer OVCAR-3 cells. Drug. Res. 67, 13-19 https://doi.org/10.1055/s-0042-115405

Manning BD, Cantley LC (2007): AKT/PKB signaling, navigating downstream. Cell 129, 1261-1274 https://doi.org/10.1016/j.cell.2007.06.009

Mao H, Lebrun DG, Yang J, Zhu VF, Li M (2012): Deregulated signaling pathways in glioblastoma multiforme, molecular mechanisms and therapeutic targets. Cancer Invest. 30, 48-56 https://doi.org/10.3109/07357907.2011.630050

Mozaffarian D, Benjamin EJ, Go AS, Arnett DK, Blaha MJ, Cushman M, Das SR, de Ferranti S, Després JP, Fullerton HJ, et al. (2016): Executive summary, heart disease and stroke statistics--2016 Update. A Report from the American Heart Association. Circulation 133, 447-454 https://doi.org/10.1161/CIR.0000000000000366

Palomero T, Dominguez M, Ferrando AA (2008): The role of the PTEN/AKT Pathway in NOTCH1-induced leukemia. Cell Cycle 7, 965-970 https://doi.org/10.4161/cc.7.8.5753

Pertovaara L, Kaipainen A, Mustonen T, Orpana A, Ferrara N, Saksela O, Alitalo K (1994): Vascular endothelial growth factor is induced in response to transforming growth factor-beta in fibroblastic and epithelial cells. J. Biol. Chem. 269, 6271-6274

Petersen M, Pardali E, van der Horst G, Cheung H, van den Hoogen C, van der Pluijm G, Ten Dijke P (2010): Smad2 and
Smad3 have opposing roles in breast cancer bone metastasis by differentially affecting tumor angiogenesis. Oncogene 29, 1351-1361 https://doi.org/10.1038/onc.2009.426

Popescu AM, Purcaru SO, Alexandru O, Dricu A (2016): New perspectives in glioblastoma antiangiogenic therapy. Contemp. Oncol. 20, 109-118 https://doi.org/10.5114/wo.2015.56122

Rickard JA, Anderton H, Etemadi N, Nachbur U, Darding M, Peltzer N, Lalaoui N, Lawlor KE, Vanyai H, Hall C (2014): TNFR1dependent cell death drives inflammation in Sharpin-deficient mice. Elife 2, 3 https://doi.org/10.7554/eLife.03464

Sang L, Coller HA, Roberts JM (2008): Control of the reversibility of cellular quiescence by the transcriptional repressor HES1. Science 321, 1095-1100 https://doi.org/10.1126/science.1155998

Schwartz GG, Steg PG, Szarek M, Bhatt DL, Bittner VA, Diaz R, Edelberg JM, Goodman SG, Hanotin C, Harrington RA, et al (2018): Alirocumab and cardiovascular outcomes after acute coronary syndrome. N. Engl. J. Med. 379, 2097-2107

Toker A, Marmiroli S (2014): Signaling specificity in the Akt pathway in biology and disease. Adv. Biol. Regul. 55, 28-38 https://doi.org/10.1016/j.jbior.2014.04.001

Wong RS (2011): Apoptosis in cancer, from pathogenesis to treatment. J. Exp. Clin. Cancer Res. 30, 87 https://doi.org/10.1186/1756-9966-30-87

Yang DH, Yoon JY, Lee SH, Bryja V, Andersson ER, Arenas E, Kwon YG, Choi KY (2009): Wnt5a is required for endothelial differentiation of embryonic stem cells and vascularization via pathways involving both Wnt/beta-catenin and protein kinase Calpha. Circ. Res. 104, 372-379 https://doi.org/10.1161/CIRCRESAHA.108.185405

Received: August 14, 2019

Final version accepted: November 5, 2019 